\title{
Diseño de un sistema interactivo orientado al usuario del museo
}

\author{
Rocío MiHURa LóPEZ \\ Universidade da Coruña \\ mihura@udc.es \\ Viviana BARNECHE NAYA \\ Universidade da Coruña \\ viviana.barneche@udc.es \\ Luis Antonio HeRnÁNDEZ IBÁÑEZ \\ Universidade da Coruña \\ lhernandez@udc.es
}

Recibido: 07/11/2012

Aceptado: 23/01/2013

\section{Resumen}

Este artículo propone la aplicación del conocimiento existente sobre el visitante del museo al diseño de los sistemas interactivos que hoy en día son habituales en este entorno, con el fin de mejorar la experiencia educativa de este usuario singular.

Palabras clave: Interactivo, diseño centrado en el usuario (UCD), usabilidad, museo, visitante.

\section{Design of an Interactive System Museum-User Oriented}

\begin{abstract}
Abastract
This paper proposes the application of existing knowledge about the museum visitor to the design of interactive systems that are common today in the museum, in order to improve the educational experience of this peculiar user.

Keywords: Interactive, User Centered Design (UCD), Usability, Museum, Visitor.

\section{Referencia normalizada}

MIHURA LÓPEZ, Rocío; BARNECHE NAYA, Viviana; y HERNÁNDEZ IBÁÑEZ, Luis Antonio (2013): "Diseño de un sistema interactivo orientado al usuario del museo". Estudios sobre el mensaje periodístico. Vol. 19. Núm. especial abril, págs.: 879-885. Madrid, Servicio de Publicaciones de la Universidad Complutense.
\end{abstract}

Sumario: 1. Introducción. 2. Metodología. 3. Desarrollo; 3.1. Desde una perspectiva museística: breve taxonomía del visitante; 3.2. Para una aplicación museística: diseño del interactivo; 3.3. En un entorno museístico: diseño del interactivo orientado al visitante del museo. 4. Conclusiones. 5. Referencias bibliográficas.

\section{Introducción}

A lo largo del siglo XX, los artilugios electrónicos comenzaron a ser un elemento familiar en el hogar medio, en forma de electrodomésticos cada vez más complejos y sofisticados, televisores con una calidad de reproducción siempre en continua progresión, equipos de música con multitud de opciones, vídeos, reproductores grabadores... La progresiva popularización de estos medios es espectacularmente veloz. Entre 1985 y 2003, el porcentaje de hogares con ordenador familiar subió del dieciocho por ciento al ochenta por ciento. En el año 2003, el sesenta y ocho por ciento de ellos estaba conectado a internet (Huysmans, et al., 2004). Esta paulatina familiariza- 
ción por parte del ciudadano con los aparatos, primero analógicos y después digitales, favorece su inclusión en el ámbito museístico, un lugar donde, por tradición, el objeto expuesto prima sobre cualquier otro elemento. Los más reticentes a la entrada de estos sistemas fueron los mismos conservadores y directores de los centros, que veían en la electrónica o medios digitales una distracción para verdadero sentido de esta institución. En palabras de Aurora León: "La utilización de estos medios extra-artísticos ha levantado serias polémicas en el seno del campo museológico, lo cual es exponente, por una parte, de la inadaptación del museo al ritmo del progreso actual y, por otra, del excesivo abuso que en ocasiones se ha hecho de estos recursos técnicos" (León, 1990, p. 101).

Los primeros dispositivos instalados en las salas de exposición eran, en su mayoría, estáticos, esto es, orientados a un espectador pasivo. Las condiciones sociales, la aparición de la cultura digital y el abaratamiento de los soportes propiciaron que los dispositivos comenzasen a ofrecer cada vez más interactividad. El público de los museos mostró un creciente interés en la visita a través de lo digital. Lo que para algunos teóricos favorece la implicación del espectador, para otros resta información a la visita, conocimiento del entorno y diluye el aura del objeto expuesto, que es la razón de ser del museo.

El sistema audio-guía y las pantallas multimedia informativas fueron los dispositivos más comunes. Ambos tuvieron aceptación por parte de los dos sectores, ya que no restaban importancia al objeto en sí y eran accesibles para todo tipo de público. Los audio-guía proporcionaban información sonora sobre los objetos expuestos, mientras que los puestos multimedia bajo la forma de pantallas adyacentes a los objetos expuestos suponían, en realidad, una actualización de las tarjetas impresas que se colocaban hasta entonces.

Los diferentes medios expositivos interactivos evolucionaron a la par que la cultura digital. Según el tipo de institución que los encarga, estos dispositivos son cada vez más especializados y abundantes, dirigidos a todo tipo de público que a su vez está familiarizado con su empleo. Los Museos de Ciencia son quizás los más proclives a su uso. A finales de los años 80 comienza a ser habitual la presencia de puestos multimedia alternos con los objetos en exposición, tanto para contextualizar la muestra como para ofrecer información alternativa o juegos educativos relacionados con el tema principal. Los estudios sobre el usuario refuerzan estos elementos al considerarlos una fuente de entretenimiento y diversión asociada al aprendizaje. Actualmente resulta extraña la visita al museo sin encontrar la presencia de lo digital, acompañando al objeto o planteando una experiencia educativa sobre lo intangible.

\section{Metodología}

El análisis se realizará desde la perspectiva de dos ramas de conocimiento. Por una parte, se acudirá a la museología clásica para obtener clasificaciones básicas sobre el visitante del museo, elaboradas por la disciplina del estudio del visitante. Por otro lado se estudiarán los elementos diferenciadores de un sistema interactivo diseñado para un museo, donde el usuario/visitante singular del museo es la base para esta diferenciación. 


\section{Desarrollo}

\subsection{Desde una perspectiva museística: breve taxonomía del visitante}

Una de las clasificaciones sobre el visitante más habitual es la realizada por Eliseo Verón y Martine Levasseur en 1983 a partir de la exposición "Vacances en France" acogida por la Bibliothèque Publique d'Information du Centre Georges Pompidou. Partiendo de grabaciones sobre el público elaboraron una clasificación de cuatro tipologías de visitante, a saber:

a) Modelo hormiga: Realiza el recorrido de forma ordenada y metódica, cronológicamente. Según Veron y Levasseur presentan valores negativos en cuanto su pasividad, con una actitud similar a la de cualquier televidente. Representa el modelo social de persona con poco nivel cultural pero con voluntad de aprender, consciente de sus carencias.

b) Modelo mariposa: Se mueven de forma pendular, su motivación es la curiosidad sobre el tema trascendiendo lo que el museo quiere mostrar en un orden concreto. Son aquellos que son conscientes de su nivel cultural y lo manejan apropiadamente.

c) Modelo pez: Recorre la exposición de forma desconfiada, dedicando poco tiempo a cada elemento y sin concentrarse realmente en el conjunto de enunciados. Agrupa al sector de personas que valoran la cultura aunque la contemplan con cierto resquemor.

d) Modelo saltamontes: Se mueve de forma similar al "pez", sin aceptar el orden predispuesto, con un recorrido libre y azaroso, solo se centra en aquellos elementos que por algún tipo de asociación despiertan su interés. Es un caso particular de grupo social que según los autores no se encuentra en otro tipo de evento cultural.

Otro estudio basado en los comportamientos observables y medibles del visitante es el realizado por Omar Calabrese en el año 2001 (Zunzunegui, 2003, p. 64). A partir de la observación directa y encuestas realizadas de forma aleatoria, obtuvo una serie de tipologías sobre las actitudes del visitante desde una taxonomía relativa al conocimiento, a saber:

a) Visitante Curioso: no sabe y quiere saber

b) Visitante Aprendiz: no sabe y debe saber

c) Visitante Competente: ya sabe y quiere saber más

d) Visitante Erudito: ya sabe y debe saber más

Para el análisis presentado en este artículo, se escogen estas dos tipologías ya que, aunque ambas se refieren a la dimensión cognitiva de la persona, combinadas permiten diferenciar al visitante del museo de cualquier otro tipo de usuario que realice un aprendizaje a partir de material educativo, en su casa o en un centro específico.

\subsection{Para una aplicación museística: diseño del interactivo}

Existen tres términos que a menudo son motivo de confusión cuando, desde la perspectiva de un museo, se define una instalación interactiva. Los términos son interactive (interactivo), hands-on (práctico o manual) y participatory (participativo). Kathleen McLean en su libro "Planning for People in Museums Exhibition" realiza 
una excelente definición que a continuación se resume. Según esta autora, el término interactivo necesita de una acción recíproca entre el visitante y la exposición; manual o práctico significa que está permitido tocar, pero no conlleva necesariamente interacción, y participativo se centra más en la capacidad de reacción del visitante que de la exposición en sí. Por lo tanto, en una exposición interactiva, el visitante puede guiar la actividad, recoger evidencias, seleccionar opciones formar conclusiones y probar sus habilidades.

En este artículo se propone que se considere una instalación interactiva a aquella que, sobre un soporte digital, exponga contenidos empleando diferentes canales, con la posibilidad por parte del usuario/os de intervenir el flujo de información adaptándolo a su demanda. Para diseñar esta nueva manera de exponer, es necesario conocer al visitante llegando más allá de las encuestas demográficas, definiendo sus posibles habilidades con lo digital, en aras de conseguir instalaciones amigables, donde lo digital resulte totalmente integrado de forma que se diluya en el entorno.

El diseño de un sistema interactivo genérico implica a varias áreas de conocimiento, que no siempre tienen en cuenta las mismas prioridades. En primer lugar se definirá la interacción desde una perspectiva genérica amplia, para después centrarse en el interactivo en el ámbito museístico, buscando en ambas definiciones al visitante como razón de ser de este diseño.

Según describe Jonas Löwgren (2013) el diseño de la interacción o de un producto interactivo tiene habitualmente dos interpretaciones diferenciadas, que sin embargo se acercan cada vez más en la práctica y en la investigación. La primera de ellas lo entiende como una disciplina de diseño centrada en la producción de elementos digitales, como software, aparatos electrónicos personales, cualquier artículo digital para consumo. Ya que se define como una rama del diseño clásico, está más cerca del diseño industrial que de la ingeniería o de las ciencias de la conducta. La otra tendencia entiende el diseño interactivo como derivado del Human Computer Interaction (en adelante $\mathrm{HCI}$ ), una disciplina que se origina en la psicología experimental y en la informática. La definición aportada por el ACM SIGCHI Curricula for Human-Computer Interaction en 1992 fue la siguiente: "Human-Computer Interaction (HCI) is a discipline concerned with the design, evaluation and implementation of interactive computing systems for human use and with the study of major phenomena surrounding them." (Hewett et al., 1997). Esta definición para el diseño de interactivos se adecúa al objeto del presente artículo, que entiende al usuario/visitante del museo como objetivo final para el diseño de estos productos.

La definición aportada por el ACM concuerda con los estudios realizados por Thomas y Mintz (1998) sobre la presencia de dispositivos multimedia en los museos. Estas autoras proponen cuatro aspectos diferenciadores para los interactivos cuando pertenecen al ámbito museo, a saber: en primer lugar está la influencia del contexto, encarnada en dos elementos: el objeto expuesto y el visitante. En el caso del objeto expuesto, los multimedia interaccionan con objetos incluidos o adyacentes a la exposición sobre los que versa el conjunto. En cuanto al visitante, éste tiene la particularidad de ser absolutamente heterogéneo (edad, nivel cultural, nivel de conocimiento informático etc.) por lo que las directrices del diseño del soporte se han de adecuar a 
una gran variedad de requerimientos. En segundo lugar Thomas y Mintz (1998) proponen el contenido del multimedia como elemento diferenciador. Los museos son instituciones educativas, esto establece una diferencia fundamental con otros multimedia cuyos contenidos se orientan más hacia el entretenimiento o la mera información. El tercer punto de diferenciación se centra en el diseño de la interfaz. Dada la variedad de público en un museo, el medio a través del cual nos comunicamos con la máquina o máquinas que conforman la instalación ha de ser prácticamente transparente, amigable y de aprendizaje cero. De nuevo el visitante establece la diferencia. En último lugar encontramos el diseño de la interacción en sí. Ya que el visitante dedica una cantidad de tiempo muy limitada a cada elemento, el interactivo debe ser claro, atractivo visualmente y ofrecer en pocos pasos la información deseada. En conclusión a este estudio se puede situar de nuevo al visitante del museo como elemento diferenciador en el diseño del interactivo cuando éste se plantea para el ámbito museo.

Ben Gammon (1999) por su parte, plantea tres cuestiones que resultan clave a la hora de diseñar un elemento interactivo para un museo. Aunque estos tres puntos fueron obtenidos tras el análisis de elementos interactivos mecánicos, no digitales, el mismo autor reconoce que las conclusiones pueden aplicarse igualmente a interactivos digitales. Gammon se concentra en tres puntos clave: la ergonomía, la comunicación con el visitante y la motivación del mismo. Las dos primeras cuestiones coinciden básicamente con Thomas y Mintz (1998); en cuanto a la motivación, Gammon responsabiliza a los elementos interactivos de la calidad de aprendizaje que el visitante adquiera. Gammon vuelve, por lo tanto, al visitante como elemento diferenciador.

Como resumen de lo anteriormente expuesto, se muestra a continuación un cuadro explicativo sobre los elementos diferenciadores de un sistema interactivo diseñado para un museo, donde el usuario/visitante singular del museo es la base para esta diferenciación.

Tabla 1: Elementos diferenciadores de un sistema interactivo diseñado para un museo. Elaboración propia

\begin{tabular}{|c|c|c|}
\hline \multicolumn{2}{|c|}{ Diseño del Interactivo en el museo: factores diferenciadores } \\
\hline \multicolumn{1}{|c|}{ Thomas y Mintz } & Gammon & FCI \\
\hline $\begin{array}{c}\text { Aspecto diferenciador 1: influencia en el con- } \\
\text { texto }\end{array}$ & $\begin{array}{l}\text { Motivación del visitante } \\
\text { en el museo }\end{array}$ & Factores ambientales \\
\hline $\begin{array}{c}\text { Aspecto diferenciador 2 y 4: diseño de conte- } \\
\text { nido educativo adaptado a un aprendizaje } \\
\text { singular }\end{array}$ & Finalidad: aprendizaje & $\begin{array}{c}\text { Finalidad variada, pero } \\
\text { siempre implementada } \\
\text { cara al usuario }\end{array}$ \\
\hline Aspecto diferenciador 3: visitante heterogéneo & Ergonomía del visitante & Uso humano \\
\hline
\end{tabular}

\subsection{En un entorno museístico: diseño del interactivo orientado al visitante del museo}

A partir de las clasificaciones sobre el visitante de museo desde una perspectiva cognitiva, realizadas por los autores anteriormente citados, se puede establecer una relación de paralelismo entre ambas y constatar cómo los sistemas multimedia interactivos, cuando están diseñados para el usuario del museo con toda su complejidad y heterogeneidad, no solo propician el aprendizaje a medida por parte de cada 
tipo de usuario, si no que además influyen positivamente en la implicación que el visitante tiene sobre su propio aprendizaje.

El modelo hormiga o visitante aprendiz, no sabe y debe saber. Los sistemas interactivos resultan atractivos y deben adaptarse a cualquier nivel de conocimiento que presente el usuario. El modelo pez o visitante curioso afronta la visita al museo de forma superficial, dedicando escaso tiempo y concentración a cada elemento, pero con voluntad de aprender. Esta aproximación a la fuente de conocimiento limitada por el propio nivel de frustración del usuario es casi específica de este entorno. El usuario no es el comisario de la exposición por lo que los contenidos que le rodean no han sido seleccionados por él mismo; sin embargo, tiene la capacidad de interrumpir a demanda el flujo de conocimiento. La calidad de la educación adquirida durante la visita se incrementará cuanto mejor sea el diseño de estos interactivos. El modelo mariposa es consciente de su conocimiento sobre el tema expuesto, aunque precisa de un orden impuesto por el comisario para la interpretación de la exposición. Los elementos interactivos no interfieren en la presentación de los objetos. El modelo saltamontes puede representar al erudito que extrae de cada visita al museo elementos de conocimiento muy particularizados. Este tipo de visitante puede encontrar en un elemento interactivo la libertad necesaria para profundizar en el conocimiento de temas muy específicos.

Todos los modelos presentan en común una dimensión fisiológica y cognitiva que un sistema interactivo, cuando está orientado al visitante del museo, puede abarcar para favorecer el aprendizaje durante la visita al museo.

\section{Conclusiones}

El diseño orientado al usuario incrementa la efectividad del producto. La aplicación del conocimiento sobre el visitante del museo al diseño de los productos interactivos incluidos en las exposiciones puede erradicar muchos problemas que éstos presentan por falta de adecuación. Es necesario singularizar al usuario del museo respecto a otros tipos de usuario de interactivo.

En algunas ocasiones puede ocurrir que el perfil de este visitante se tenga en cuenta de manera exclusivamente teórica, sin ningún tipo de participación activa. Como línea para futuras investigaciones en este campo, se está trabajando en la aplicación de la metodología del diseño centrado en el usuario con especial atención a la inclusión del usuario en el ciclo vital de diseño del producto.

\section{Referencias bibliográficas}

BAILEY, Robert W. (1982): Human Performance Engineering: Guide for System Designers. NJ, Prentice-Hall.

GAMMON, Ben (1999): "Everything we currently know about making visitorfriendly mechanical interactive exhibits". Informal Learning, n 39,10-13 Nov-Dec

HEWETT Thomas T. et al (1997): “ACM SIGCHI Curricula for Human-Computer Interaction". [En línea] en ACM Special Interest Group on Computer human Interaction: http://sigchi.org/cdg/cdg2.html\#2_1 [fecha de consulta: 30 junio 2009] 
HUYSMANS, Frank; DE HAAN, Jos \& VAN DEN BROE, Andries (2004): Achter de schermen. Sociaal en Cultureel Planbureau. http://www.scp.n1/Publicaties /Alle publicaties/Publicaties 2004/Achter de schermen

LEÓN, Aurora (1990): El museo. Teoría, praxis y utopía. Madrid, Cátedra.

LÖWGREN, Jonas (2013): "Interaction Design-brief intro". In: SOEGAARD, Mads and DAM, Rikke Friis (eds.). "The Encyclopedia of Human-Computer Interaction, 2nd Ed.". Aarhus, Denmark: The Interaction Design Foundation. http://www.interaction-design.org/encyclopedia/interaction_design.html

THOMAS, Selma \& MINTZ, Ann (eds., 1998): The Virtual and the Real. Media in the Museum. Washington DC, American Assiciaton of Museums.

ZUNZUNEGUI, Santos (2003): Metamorfosis de la mirada. Museo y Semiótica. Madrid, Cátedra.

\section{Rocío MIHURA LÓPEZ}

VideaLAB. Universidade da Coruña

Profesora en la Facultade de Ciencias da Comunicación

mihura@udc.es

\section{Viviana BARNECHE NAYA}

VideaLAB. Universidade da Coruña

Facultade de Ciencias da Comunicación

Arquitecta, docente en el Máster en Creación y Comunicación Digital

viviana.barneche@udc.es

\section{Luis Antonio HERNÁNDEZ IBÁÑEZ}

VideaLAB. Universidade da Coruña

Doctor Arquitecto. Director del Grupo de Visualización Avanzada en Arquitectura, Ingeniería Civil y Urbanismo - VideaLAB

Profesor Titular en la Escuela Técnica Superior de Ingenieros de Caminos, Canales y Puertos; y de la Facultad de Ciencias de la Comunicación de la Universidad de A Coruña

lhernandez@udc.es 